\title{
THE SPECTRAL AND SPATIAL DECOMPOSITION OF EXTRAGALACTIC RADIO SOURCES
}

\author{
L. RUDNICK ${ }^{1}$ AND D.M. KATZ-STONE ${ }^{1,2}$ \\ ${ }^{1}$ University of Minnesota - Dept. of Astronomy 116 Church St. \\ SE, Minneapolis, MN 55455 USA \\ 2 U.S. Naval Academy 572 Holloway Road, Annapolis, MD \\ 21402-5026 USA
}

We have developed a new set of techniques for extracting information from maps of synchrotron emission. Some of these methods are used to determine the shape of the spectrum and then to isolate the contributions of the number and energy of relativistic electrons and the local magnetic field strength to the total intensity images. These are discussed in KatzStone, Rudnick \& Anderson (1993), Katz-Stone \& Rudnick (1994), and Rudnick, Katz-Stone \& Anderson, (1994). Here, we describe a method called spectral tomography - to remove confusion from various features along the line of sight. We find that both structural and spectral confusion are commonplace, and therefore our dynamical and radiative models of extragalactic sources need significant revision.

To conduct a tomography analysis, start with two matched maps $B_{\nu 1}(x, y)$ and $B_{\nu 2}(x, y)$. Then, form a set of tomography maps:

$$
B_{t}(x, y) \equiv B_{\nu 1}(x, y)-t \times B_{\nu 2}(x, y)
$$

for a range of values of $t$. Suppose now that we have a feature, A, with a spectral index $\alpha_{A}$. For the tomography map where $\alpha_{t} \equiv \log (t) / \log \left(\frac{\nu 1}{\nu 2}\right)=$ $\alpha_{A}$, the feature A will then completely disappear from the map. If another feature is along the same line of sight with $\mathrm{A}$, and has a different spectral index, then it can be seen without confusion (for Cygnus A results, see Rudnick \& Katz-Stone, 1996).

The figure below shows part of the spectral tomography analysis for the wide-angle-tail source $1231+674$. Each line represents a slice at the same 
declination in the southern tail, for a series of tomography maps $B_{t}$. In the top slice, we see a broad peaked feature commonly associated with the flaring jet. In the middle slices, corresponding to a tomography spectral index $\approx 0.5$, the central peaked feature disappears, leaving a broader, flattopped envelope. Below this, the central feature goes negative while the envelope remains positive. The tomography maps show that this behavior is due to a flatter spectrum jet surrounded by a steeper spectrum sheath.

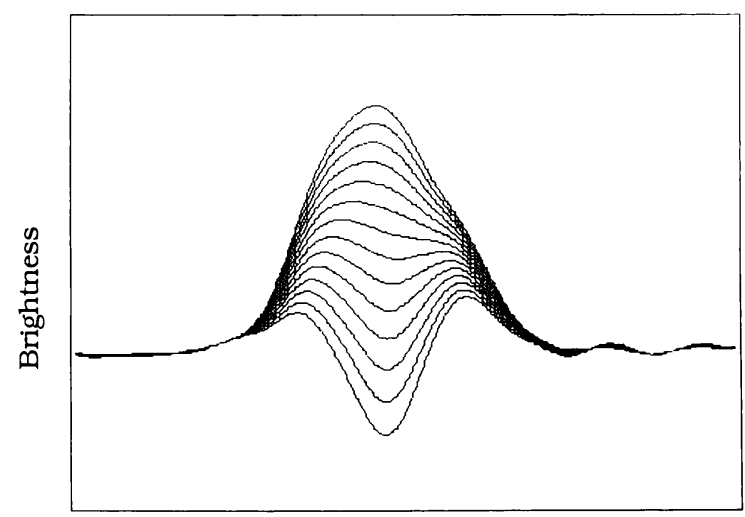

We find similar features in $3 \mathrm{C} 449$ and another WAT, 1433+553. After separating the jet from the sheath, there is little or no spectral aging of the jets, even though they appear to age when confused with the steep spectrum sheaths. This raises both dynamical questions regarding the origins of the sheaths, (e.g., turbulent layers - Bicknell, 1994 or two-component outflows - Sol, Pelletier \& Asseo, 1989), and a need for new aging analyses. Our tentative conclusion is that adiabatically shifted curved electron populations and magnetic fields may dominate the effects we commonly call aging.

ACKNOWLEDGMENTS. This work is supported in part at the University of Minnesota through US NSF grant AST93-18959, using data obtained at the Very Large Array, a facility of the NRAO, operated by AUI under contract with the U.S. NSF. We appreciate the sharing of data by A. O'Donoghue $(1231+674,1433+553)$, L. Feretti and R. Perley (3C449) and good discussions from too many people to fit here.

\section{References}

Bicknell, G.V., 1994, In ASP Conference Series, (ed. G. Bicknell, M. Dopita, \& P. Quinn) Vol. 54, 357, ASP.

Katz-Stone, D.M., Rudnick, L. and Anderson, M.C. (1993), Ap. J., 407, 544

Katz-Stone, D.M. \& Rudnick, L., 1994, Ap. J., 426, 116.

Rudnick, L., Katz-Stone, D.M. \& Anderson, M.C., 1994, Ap. J. Suppl. Ser., 90, 955.

Rudnick, L. \& Katz-Stone, D.M., 1996, In Green Bank Workshop on Cygnus A, (ed. C. Carilli \& D. Harris),58, Cambridge University Press

Sol, H., Pelletier, G., \& Asseo, E., 1989, M.N.R.A.S., 237, 411 